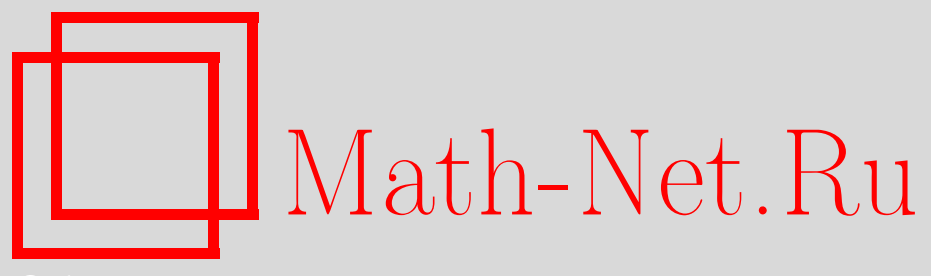

В. Г. Звягин, А. В. Кузнецов, О плотности множества правых частей начально-краевой задачи модели Джеффриса с объективной производной Яуманна, УМH, 2008, том 63, выпуск 6, 165-166

DOI: https://doi.org/10.4213/rm9249

Использование Общероссийского математического портала Math-Net.Ru подразумевает, что вы прочитали и согласны с пользовательским соглашением http://www . mathnet.ru/rus/agreement

Параметры загрузки:

IP : 54.198 .55 .26

26 апреля 2023 г., 04:15:55

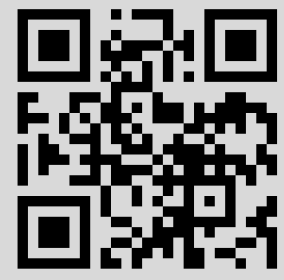




\section{О плотности множества правых частей начально-краевой задачи модели Джеффриса с объективной производной Яуманна}

\section{В. Г. Звягин, А. В. Кузнецов}

Рассматривается следующая начально-краевая задача, описывающая движение вязкоупругих сред типа жидких растворов полимеров, битумов, бетона, земной коры (модель Джеффриса с объективной производной Яуманна):

$$
\begin{gathered}
\frac{\partial v}{\partial t}-\mu_{1} \Delta v+\sum_{i=1}^{n} v^{i} \frac{\partial v}{\partial x_{i}}+\nabla p-\operatorname{Div} \tau=f, \\
\frac{\partial \tau}{\partial t}+\sum_{j=1}^{n} v^{j} \frac{\partial \tau}{\partial x_{j}}+\tau W(v)-W(v) \tau+\frac{1}{\mu_{2}} \tau=2 \mu_{3} \mathscr{E}(v), \\
\operatorname{div} v(t, x)=0, \quad(t, x) \in[0, T] \times \Omega,\left.\quad v\right|_{[0, T] \times \partial \Omega}=0, \\
\left.v\right|_{t=0}=v_{0},\left.\quad \tau\right|_{t=0}=\tau_{0}, \quad \int_{\Omega} p(t, x) d x=0 .
\end{gathered}
$$

Здесь $\Omega \subset \mathbb{R}^{n}$ - ограниченная область с границей класса $C^{\infty}, n=2,3,(t, x) \in$ $[0, T] \times \Omega, v(t, x)=\left(v^{1}(t, x), \ldots, v^{n}(t, x)\right)$ - поле скоростей, $p(t, x)$ - давление среды, $\mathscr{E}(v)=\left(\mathscr{E}_{i j}(v)\right)_{j=1, \ldots, n}^{i=1, \ldots, n}-$ тензор скоростей деформации, $W(v)=\left(W_{i j}(v)\right)_{j=1, \ldots, n}^{i=1, \ldots, n}-$ тензор завихренности, $\tau=\left(\tau_{i j}\right)_{j=1, \ldots, n}^{i=1, \ldots, n}=\sigma-2 \mu_{1} \mathscr{E}(v)$ - упругая компонента тензора напряжений $\sigma, \operatorname{Div} \tau-$ вектор с координатами $(\operatorname{Div} \tau)_{j}=\sum_{i=1}^{n} \frac{\partial \tau_{i j}}{\partial x_{i}}, \mu_{1}, \mu_{2}, \mu_{3}-$ некоторые положительные константы, имеющие физический смысл, $f$ - плотность внешних сил, действующих на среду. Плотность среды считается равной единице.

Опишем используемые функциональные пространства и операторы. Пусть $H-$ замыкание пространства $\mathscr{V}=\left\{u \in C_{0}^{\infty}\left(\Omega, \mathbb{R}^{n}\right): \operatorname{div} u=0\right\}$ в пространстве $L_{2}\left(\Omega, \mathbb{R}^{n}\right)$ и $P: L_{2}\left(\Omega, \mathbb{R}^{n}\right) \rightarrow H-$ проектор Лере. Известно (см., например, [1]), что оператор $-P \Delta: H^{2}\left(\Omega, \mathbb{R}^{n}\right) \rightarrow H$, где $\Delta$ - оператор Лапласа, продолжается до замкнутого самосопряженного положительно определенного оператора в $H$, который будем обозначать через $A$ и собственные функции $\left\{e_{j}(x)\right\}_{j=1}^{\infty}$ которого образуют ортонормированный базис в $H$. Пусть $0<\lambda_{1} \leqslant \lambda_{2} \leqslant \cdots \leqslant \lambda_{k} \leqslant \cdots$ - собственные значения оператора $A$, т. е. $A e_{j}=\lambda_{j} e_{j}, j \in \mathbb{N}$. Обозначим $E_{\infty}=\left\{v: v=\sum_{j=1}^{m} v_{j} e_{j}, v_{j} \in \mathbb{R}, m \in \mathbb{N}\right\}$. Определим норму $\|\cdot\|_{\alpha}, \alpha \in \mathbb{R}$, равенством $\|v\|_{\alpha}=\left(\sum_{j=1}^{\infty} \lambda_{j}^{\alpha}\left|v_{j}\right|^{2}\right)^{1 / 2}$. Следуя [1], обозначим символом $V^{\alpha}, \alpha \in \mathbb{R}$, пространство, являющееся пополнением $E_{\infty}$ по норме $\|\cdot\|_{\alpha}$. Отметим, что $V^{0}=H$. Пусть $M_{\mathrm{s}}(n)$ - пространство симметричных матриц порядка $n$ и $\mathscr{H}^{\alpha}=H^{\alpha}\left(\Omega, M_{\mathrm{s}}(n)\right)$ - соболевское пространство функций со значениями в $M_{\mathrm{s}}(n)$. Положим $X=\left\{u \in L_{2}\left(0, T ; V^{3}\right): u^{\prime} \in L_{2}\left(0, T ; V^{-3}\right)\right\}$ и обозначим через $F$ множество правых частей $f \in L_{2}(0, T ; H)$ задачи (1)-(4), для которых существует решение $(v, \tau) \in X \times C\left([0, T] ; \mathscr{H}^{2}\right)$.

ОСНовной РезУЛЬтат. Пусть $v_{0} \in V^{3}, \tau_{0} \in \mathscr{H}^{2}$. Тогда $F \subset L_{2}(0, T ; H)$ всюду плотно относительно топологии пространства $L_{p}\left(0, T ; V^{-l}\right)$, где l u p удовлетворяют одному из следующих условий:
1) $l \geqslant 6, p=\infty$;
2) $6>l \geqslant 3, p=2$.

Работа выполнена при поддержке РФФИ (гранты № 07-01-00137, 08-01-00192). 
Схема ДоказАтельствА. В лемме 2.3 работы [2] показано, что для любого $v \in$ $L_{1}\left(0, T ; V^{3}\right)$ существует и единственно решение $\tau=M(v) \in C\left([0, T] ; \mathscr{H}^{2}\right)$ задачи (2) с начальным условием $\left.\tau\right|_{t=0}=\tau_{0}$. Нам необходимо представление $\tau$ через $v$. Для его получения перепишем (2) в эквивалентной интегральной форме:

$$
\tau(t, x)=e^{-t / \mu_{2}} \tau_{0}(z(0, t ; x))+\int_{0}^{t} e^{(s-t) / \mu_{2}}\left(2 \mu_{3} \mathscr{E}(v)-\tau W(v)+W(v) \tau\right)(s, z(s, t ; x)) d s,
$$

где $z(s, t ; x)=x+\int_{t}^{s} v(\xi, z(\xi, t ; x)) d \xi$. Далее, положим $C_{D}^{1}(\bar{\Omega})=\{z: \bar{\Omega} \leftrightarrow \bar{\Omega}:$ $\left.\left.z\right|_{\partial \Omega}=I, z \in C^{1}\left(\bar{\Omega}, \mathbb{R}^{n}\right), \forall(x \in \Omega) \operatorname{det}\left|\frac{\partial z}{\partial x}(x)\right|=1\right\}$. Обозначим $C G^{1}=C([0, T] \times$ $\left.[0, T] ; C_{D}^{1}(\bar{\Omega})\right)$ с естественной топологией. Тогда определен оператор $Z: X \rightarrow C G^{1}$, $Z(v)(s, t ; x)=z(s, t ; x)$, являющийся вполне непрерывным. Обозначим через $Q: X \times$ $C G^{1} \rightarrow L_{2}\left(0, T ; \mathscr{H}^{1}\right)$ оператор, определяемый соответствием $(v, z) \mapsto \theta$, где $\theta$ - решение уравнения (5). Отметим, что оператор $M$ допускает диагональное представление $M(v)=Q(v, Z(v))$.

Рассмотрим следующее $\delta$-возмущение (с помощью оператора $\delta A^{3}$ ), $\delta>0$, операторного уравнения, соответствующего задаче (1)-(4):

$$
L_{\delta} v+K(v)+G(v)-\left(f, v_{0}\right)=0,
$$

где операторы $L_{\delta}, K, G: X \rightarrow L_{2}\left(0, T ; V^{-3}\right) \times V^{0}$ определяются равенствами: $L_{\delta} v=$ $\left(v^{\prime}+\mu_{1} A v+\delta A^{3} v,\left.v\right|_{t=0}\right), K(v)=\left(P \sum_{j=1}^{n} v^{j} \frac{\partial v}{\partial x_{j}}, 0\right), G(v)=(-P \operatorname{Div} Q(v, Z(v)), 0)$. Справедливы следующие утверждения.

Теорема 1. Для всех решений $v \in X$ уравнения (6) при $\delta \geqslant 0$ имеет место априорная оценка:

$$
\|v\|_{L_{\infty}\left(0, T ; V^{0}\right)}^{2}+\mu_{1}\|v\|_{L_{2}\left(0, T ; V^{1}\right)}^{2}+2 \delta\|v\|_{L_{2}\left(0, T ; V^{3}\right)}^{2} \leqslant \frac{2}{\mu_{1}}\|f\|_{L_{2}\left(0, T ; V^{-1}\right)}^{2}+2\left\|v_{0}\right\|_{0}^{2} .
$$

Теорема 2. Уравнение (6) имеет решение в $X$ при любых $\delta>0$.

Из теоремы 2 , оценки (7) и того, что $\left\|\delta A^{3} v\right\|_{L_{\infty}\left(0, T ; V^{-6}\right)} \leqslant \delta\|v\|_{L_{\infty}\left(0, T ; V^{0}\right)} \rightarrow 0$ при $\delta \rightarrow 0$ и $\left\|\delta A^{3} v\right\|_{L_{2}\left(0, T ; V^{-3}\right)}=\sqrt{\delta}\|\sqrt{\delta} v\|_{L_{2}\left(0, T ; V^{3}\right)} \rightarrow 0$ при $\delta \rightarrow 0$, следует основной результат работы.

Соответствующий результат для системы Навье-Стокса см. в [1].

\section{Список литературы}

[1] А.В. Фурсиков, Оптимальное управление распределительными системами. Теория и приложения, Научная книга, Новосибирск, 1999; англ. пер.: A. V. Fursikov, Optimal control of distributed systems. Theory and applications, Transl. Math. Monogr., 187, Amer. Math. Soc., Providence, RI, 2000. [2] C. Guillopé, J.-C. Saut, Nonlinear Anal., 15:9 (1990), 849-869.

\section{В. Г. Звягин (V. G. Zvyagin)}

Воронежский государственный университет E-mail: zvg@main.vsu.ru
Представлено В. М. Бухштабером Принято редколлегией 05.10 .2008

\section{А. В. Кузнецов (А. V. Kuznetsov)}

Воронежский государственный университет

E-mail: kuznetsov@math.vsu.ru 